\title{
PNEUMATIC SEPARATION OF COFFEE BERRIES
}

A pneumatic cleaner and separator has been adapted to sort freshly picked coffee fruit. This equipment virtually eliminates need for hand labor for this operation.

An inlet conveyor feeds approximately 45 almudes $^{1}$ of berries per hour into a stream of air which divides the berries into a light and a heavy fraction. The velocity of the air stream is adjusted to the point where the heavy fraction contains less than 10 percent of green fruit. A moving air stream selects by a combination of density and drag force, the latter being propor. tional to the cross-sectional area of the individual berries. The heavy fraction therefore will contain both larger and heavier fruit.

The heavy fraction in laboratory tests contained 80 to 90 percent of the berries, was virtually free of undeveloped, small-green, overripe and dried berries, and could be pulped and processed by the wet method to satisfy first-quality, grade standards. The light fraction contained dead, undevelopoped, immature, damaged berries as well as a small fraction of ripe berries. These were mostly single seed berries. This fraction was dried as whole berries by the dry method.

Leaves are held against the air escape mesh with most trash until the blower is turned off. The leaves then fall and can be collected separately with the trash. If this machine is used, a soak tank for berries is not required. Hence, berries that would float are not mixed with the trash and there is no difficulty in recovering. Also, because a dry separation is made, pickers can be rewarded and outside purchases of berries assessed on the basis of the weight of the heavy fraction, thus on quality, rather than by total weight or volume.

\author{
D. J. van Rest \\ Department of Agricullural Engineering
}

${ }^{1}$ An almud is equivalent to about 28 pounds of coffee berries. 